\title{
La gestión patrimonial desde la periferia: el arte contemporáneo como regenerador del espacio rural
}

\author{
VICTORIA QUIROSA GARCÍA' \\ ORCID: 0000-0001-7512-5754 \\ vquirosa@ujaen.es
}

Patrimonial management in the periphery; contemporary art regenerating the rural fringe

\begin{abstract}
RESUMEN
Pensar en el medio rural es reflexionar sobre la tradición y modos de vida extintos. En este texto queremos analizar la reinvención de algunos núcleos rurales periféricos, como a través de la llegada y aceptación de experiencias de arte contemporáneo han sabido crear un recurso sostenible que los diferencia y que los vuelve a situar como un reclamo turístico. Analizaremos con los testimonios de gestores y artistas, la historia de esta transformación y aprendizaje que abarca iniciativas muy variadas, casos como la de Montalbán en Córdoba, Genalguacil en Málaga o Fanzara en Castellón, entre otros. Queremos reflexionar también sobre los efectos colaterales de este fenómeno, la creación de nuevas identidades o la reivindicación y puesta en valor de las expresiones culturales del territorio, modos de vida y tradición oral, en convivencia o disidencia con la implantación de estos nuevos usos del territorio.
\end{abstract}

Palabras clave: Periferia, rural, arte contemporáneo, sostenibilidad.

\section{Abstract}

To think about the rural population is to think about the tradition and extinct manners of life. In this text we want to analyze the reinvention of some rural peripheral villages, through the arrival and acceptance of experiences of contemporary art they have could create a sustainable resource that differentiates them and that turns them to placing as a tourist claim. We will analyze with the testimonies of art managers and artists the history of this transformation and learning that includes very varied initiatives, examples as Montalbán (Cordova), Genalguacil (Malaga) or Fanzara (Castellón) among others. We want to reflect on the collateral effects of this phenomenon, the creation of new identities or the recoveryin order to enhance the cultural expressions of the territory, manners of life and oral tradition, in conviviality or dissent with the introduction of these new uses of the territory. Keywords: periphery, rural, contemporary art, sustainability.

1 Universidad de Jaén. Departamento de Patrimonio Histórico. Área de Historia del Arte. 


\section{INTRODUCCIÓN: ¿DE QUÉ HABLAMOS CUANDO HABLAMOS DE ARTE?}

Hablar sobre arte en el siglo XXI implica el reconocimiento de parámetros que distan del propio fenómeno y que han modificado notablemente la imagen que tiene la sociedad sobre sus manifestaciones artísticas. Hoy día es innegable que el arte es una potente herramienta de comunicación, como lo fue desde sus inicios, pero que está supeditada en muchos casos por el valor económico que se traduce,en definitiva,en el número de visitantes, en el merchandising, en las ventas, etc. No es algo extraño, ya que el propio término patrimonio lleva implícito en su origen etimológico el carácter económico². David Lowenthal en su obra, "El pasado es un país extraño", ya reflexionaba sobre los riesgos de la sacralización de lo pretérito que lastran el presente: «Si el pasado es un país extraño, la nostalgia lo ha hecho "el país extraño con el mercado turístico más saneado de cuantos existen", ahora bien, al igual que otros turistas, aquellos que van al pasado ponen en peligro el objeto de su búsqueda». (Lowenthal, 2010, p. 29)

El arte ya no ocupa un papel central como objeto reverenciable de propaganda político-religiosa. Debe llegar a todos los ámbitos y estratos de la población porque es testimonio vivo de nuestra manera de pensar y vivir el momento presente. Hoy día, si hablamos de arte, en general, encontramos dos formas estandarizadas dirigidas a un público especializado, la primera, y a un público amateur, la segunda. Los medios a través de los cuales se conoce y se difunde el fenómeno artístico también difieren. Por un lado, el mundo académico focalizado en las Universidades e Instituciones especializadas del sector, se resiste con frecuencia a impulsar de igual forma el conocimiento del pasado y del presente, sólo tenemos que consultar los planes de estudio de nuestros grados en Historia del Arte, la escasez de tiempo y el volumen de los temarios ratifican lo dicho. La Historia del Arte sigue alimentando un modelo decimonónico abanderado por la tríada, arquitectura, pintura y escultura, que ensalza el arte figurativo y que establece jerarquías en función de estos parámetros.

Nicolás Bourriaud afirmaba en las bases fundacionales de la estética relacional:

«Los historiadores del arte están a merced de dos peligros mayores: el primero es el idealismo, que consiste en concebir el arte como un dominio exclusivamente autónomo regido por sus leyes propias. Dicho de otra manera y retomando lo expresado por Althusser, el arte es concebido como un tren del que se conocería el punto de partida, el destino y las etapas del viaje (...)». (Bourriaud, 2008, pp.80-81)

La formación actualizada tiende a ser autodidacta, Duchamp, cuyas obras tienen más de cien años sigue siendo el artista revolucionario que causa estupor, sin duda, no podríamos entender el arte contemporáneo actual sin sus aportaciones pero en estos cien años han pasado muchas cosas. Los museos y centros de arte también inciden directa-

2 http://dle.rae.es/srv/fetch?id=SBOxisN 
mente en nuestra formación artística, los más visitados y de mayor prestigio se sitúan en grandes ciudades. El turismo cultural se ha convertido en un referente en nuestro país, con casos paradigmáticos como el de Málaga, que ha experimentado un cambio radical en la última década, en la imagen que ofrece a sus visitantes. El turismo de sol y playa, un tanto demonizado, convive con un turismo masivo de crucero pero cultural ${ }^{3}$. En este texto, queremos reflexionar sobre el papel del arte actual, de la creación contemporánea, en los núcleos periféricos, aquellos alejados de los grandes museos y exposiciones temporales multitudinarias, los que han visto despobladas sus casas y calles, los que han apostado por el arte contemporáneo como herramienta de cambio social y económico.

El imaginario colectivo ha creado una visión estática del arte contemporáneo, que parte del desconocimiento y la ausencia de familiaridad, lo que dificulta eliminar una barrera que han promovido las instituciones del sector. Nuestros museos se han convertido en un producto más que ofrecer al visitante y en nuestras ciudades proliferan las franquicias como signo de prestigio. Accedemos al producto final y no al proceso, lo que dificulta nuestra comprensión de la obra, por eso, en la segunda mitad del siglo XX se puso de manifiesto que el arte podía dirigirse hacia otras metas que aunque minoritarias le permitían escapar del férreo sistema institucional. Son muy interesantes las reflexiones de los artistas Dora García e Isidoro Valcárcel, que en una entrevista afirmaban:

Isidoro Valcarcel Medina, sobre los museos: «La institución museística, por ejemplo. Se mide por el número de visitantes y se mantiene por eso. Cuantos más, mejor, claro. Los visitantes ven anuncios de exposiciones por la calle y van al museo. A otros que no se anuncian tanto pues no va nadie, porque no están "suficientemente institucionalizados". Es un organismo que cuenta con una red de colaboradores externos que están tan institucionalizados como los que dirigen la oficina». (Espejo, 2018s.p.)

Dora García: «Hace poco hablaba con un experto en instituciones y me decía que, en realidad, no se trata tanto de acabar con la institución, sino de crear muchas otras que se contrapongan, instituciones pequeñas, de tres o cuatro personas. Lo malo es esa institución total que lo decide todo sobre la vida de la gente. Aunque la institución puede ser un refugio. Estar “desinstitucionalizado” crea ansiedad». (Espejo, 2018 s.p.)

En relación al sistema institucional del arte, lo interesante no es abolirlo sino crear una estructura más flexible en la que tengan cabida un mayor número de experiencias, es decir, una diversificación del sector, en igualdad de oportunidades. Lo que requiere especialización y mediación para que el arte llegue a toda la población y espacios no jerarquizados, es en este contexto, en el que la calle, como veremos, se convierte en el espacio artístico por excelencia.

3 Consejería de Turismo y Deporte de la Junta de Andalucía. Estadísticas de Turismo, enero-agosto 2018. http://www.juntadeandalucia.es/turismoydeporte/opencms/estadisticas-consejeria/turismo/ultimosdatos/ 


\title{
2. CENTRO Y PERIFERIA, DEL OLVIDO A LA INNOVACIÓN
}

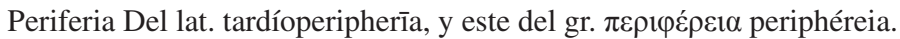 \\ 1. f. Contorno de un círculo, circunferencia. \\ 2. f. Término o contorno de una figura curvilínea. \\ 3. f. Parte de un conjunto alejada de su centro, especialmente la de una ciudad ${ }^{4}$.
}

El sábado, 30 de septiembre de 1967, Robert Smithson compró un billete de ida a Passaic, inició un viaje a la periferia que cambiaría el devenir del arte. Una acción azarosa, de igual forma que las derivas surrealistas, pero que en este caso tenía una clara intención, reivindicar la periferia a través de sus monumentos, vestigios industriales olvidados que gracias a su relato pasaban a la primera línea. «¿Qué puedes encontrar en Passaic que no puedas encontrar en París, Londres o Roma? Búscalo tú mismo. Descubre (si te atreves) el maravilloso río Passaic y los eternos monumentos que hay en sus encantados bancales. Vete en un confortable coche de alquiler hasta esas tierras perdidas por el tiempo (...)». (Careri, 2014, p.134)

Esta experiencia contagió a otras, y poco a poco se fue definiendo en primer lugar la desmaterialización del objeto artístico, una acción podía constituir la obra; en segundo lugar la interacción como eje fundamental del proceso, cuestión que trataremos a continuación, y sin duda, la presencia activa del medio que nos rodea, urbano o periférico, que es el protagonista esencial. En las décadas de los sesenta y setenta la naturaleza se convierte en el marco de acción: «En Estados Unidos esto fue bautizado como Land Art o Earthworks (...) para Simón Marchan, las poéticas vinculadas a la naturaleza que se desarrollaron en Estados Unidos no fueron sino la réplica americana del Arte Povera europeo», aunque según Pilar Parcerisas éste último otorga al objeto una mayor importancia. En España, «el paisaje y la naturaleza proporcionaron la salida al exterior, la abolición del concepto de taller, la confrontación del "yo" con la naturaleza». (Parcerisas, 2007, p.75) Dentro de esta línea también la autora define los«Conceptualismo (s) periféricos: "es un término usado a partir de los años noventa ante los estudios de las periferias del arte y del modo en que estas poéticas de ruptura se manifestaron». (2007, p.13)

El ámbito de las experiencias que vamos a analizar es la periferia, esencialmente municipios que tienen una fuerte vinculación rural agropecuaria. Apostando por la redefinición de su imagen cultural a través de la creación contemporánea. Es por todo esto que un gran número de las experiencias estudiadas se pueden definir dentro de lo que Nicolás Bourriaud denominó «Arte relacional: Conjunto de prácticas artísticas que toman como punto de partida teórico y práctico el conjunto de las relaciones humanas y su contexto social, más que un espacio autónomo y privativo» Íntimamente ligado a la «Estética relacional: "Teoría estética que consiste en juzgar las obras de arte en función 
de las relaciones humanas que figuran, producen o suscitan» (Bourriaud, 2008, p.142) En ambas, la obra de arte adquiere según este autor, un carácter ilimitado basado en la experimentación e intercambio continuo que suscita. El arte se define como un estado de encuentro en el que la obra sólo será tal con la participación del público que la hace suya: «una obra puede funcionar como un dispositivo relacional que contiene cierto grado de variables aleatorias, una máquina que provoca y administra los encuentros individuales o colectivos» (2008, p.33) Nos interesa especialmente este tema porque traslada al espectador, tradicionalmente pasivo, la obligación de participar de forma activa para que la obra cumpla su objetivo.

Partimos de la capacidad comunicativa e interactiva del arte, cuestión en la que ahonda la obra de Eugenio Ampudia: «Los artistas tenemos un papel distinto al del ciudadano normal. Pero yo, cuando creo arte, estoy sacando conclusiones políticas y estéticas». (Díaz 2015s.p.) En diversas entrevistas pone de manifiesto el papel poderoso que puede tener la obra artística: «El arte es, en sí mismo, un arma poderosa de comunicación y capaz de plantear las preguntas más incómodas, también, las más adecuadas». (Achiaga, 2015s.p.)

El Arte contemporáneo dentro de estas coordenadas se convierte en un aliado para poner en valor, comunicar y generar nuevos lazos identitarios entre la población y las obras que pueden llegar a ser colaborativas. Indudablemente, de esta forma se reafirma que el arte tiene un compromiso social y político innegable, en esta línea el artista Isidro López-Aparicio profundiza en el tema tanto en su libro, "Arte político y compromiso social. El Arte como transformación creativa de conflictos", como a través de su obra, que analizaremos en los siguientes epígrafes. En este sentido afirma:

\footnotetext{
«Todo arte es inevitablemente social y político. Cualquier obra se genera y muestra desde el testigo de la realidad social donde se ubica material o conceptualmente, sea el artista más o menos consciente de tal condición. Cada acción artística conforma tejido sociopolítico. Aunque su finalidad declarada fuera la de operar únicamente en la complacencia (...)». (López, 2016, p.9)
}

\subsection{Nuevas identidades patrimoniales en el medio rural}

La definición de conceptos trascendentales no es un acto estático, hoy día, sobre todo en el ámbito cultural, es la sociedad quien redefine y transforma los aspectos esenciales de su cotidianeidad, ratificados grupalmente.Por tanto, para que se produzca un cambio necesitamos que exista la voluntad de cambio y que sea compartida por un grupo lo más amplio posible. En ese sentido, en el ámbito que tratamos, los habitantes de pequeños municipios serán los que acepten la llegada de nuevas propuestas que con el paso del tiempo aspiran a formar parte de su identidad local. Este proceso no es sencillo y sobre todo dependerá del papel que se les otorgue, el museólogo George Henri Riviere en su definición de Ecomuseo nos hablaba de la creación de estos lazos identitarios: 


\begin{abstract}
«Un espejo, donde la población se contempla para reconocerse, donde busca la explicación del territorio en el que está enraizada y en el que se sucedieron todos los pueblos que la precedieron, en la continuidad o discontinuidad de las generaciones. Un espejo que la población ofrece a sus huéspedes para hacerse entender mejor, en el respeto de su trabajo, de sus formas de comportamiento y de su intimidad». (Rivière, 1985, p.182)
\end{abstract}

Sin duda, uno de los valores vertebradores del patrimonio cultural es el de identidad, aquello que nos define y diferencia del resto y que aúna tanto manifestaciones materiales como inmateriales, siendo estas últimas las primeras en desaparecer con la pérdida de habitantes. Muchos de nuestros municipios no atesoranun patrimonio cultural distintivo, ni están situados en enclaves únicos y es por eso que no han sido foco de atención del turismo, es en estos casos en los que nos parece interesante que incorporen nuevos valores identitarios. Desde la UNESCO, Bernard M. Feilden, Jukka Jokilehto o Herb Stovel, entre otros, han desarrollado numerosos estudios aplicados al Patrimonio Mundial, estos autores establecen que los valores identitarios son esencialmente inmateriales y aceptados grupalmente:

«Valores de identidad (basados en el reconocimiento) Relacionados con los lazos emocionales de la sociedad hacia los objetos y sitios específicos que incluyen características como: edad, tradición, continuidad, conmemoración, leyenda. También pueden ser sentimentales, espirituales, religiosos, simbólicos, patrióticos o nacionalistas. Al ser considerados como emocionalmente perceptivos, estos valores tienen un fuerte impacto en su salvaguarda, conservación y restauración». (Feilden, Jokilehto, 2003, p.28)

Pero ¿qué ocurre cuando esta herencia cultural no genera ingresos económicos? En este sentido fiestas locales que en origen eran minoritarias se han convertido en un reclamo internacional, como la Tomatina de Buñol, una fiesta que aunque comienza en 1945, será conocida en nuestro país a través de la retransmisión de un reportaje en Informe Semanal en la década de los ochenta lo que hizo crecer exponencialmente el número de participantes, para llegar a ser reclamo internacional en la última década ${ }^{5}$. Evidentemente, su castillo o su iglesia no consiguen la misma afluencia de visitantes. Siguiendo a Antonio Bellido Blanco, la Tomatina sería un claro ejemplo de creación de nuevos recursos turísticos que vienen a paliar el desinterés o el creciente abandono de municipios poco poblados: «Existen tiempos y lugares en los que la identidad no reside más que en los mitos, las creencias y los relatos, pero también en sus cosechas y el ganado y en sus vajillas y en comportamientos colaborativos». (Bellido, 2018, p.157)

\title{
2.2. Claves para el desarrollo rural sostenible
}

La despoblación rural es uno de los problemas que afecta a regiones como Castilla y León, Castilla la Mancha o La Rioja, entre otras, son datos que han salido a la luz en los 
últimos diez años al hilo de la crisis económica. Un informe publicado hace unos meses recogía como España tiene 1.840 municipios rurales en riesgo de desaparición.Esta cifra se extrae de la investigación del Centre d'EstudisDemogràfics (CED) de la UAB, realizado por Joaquín Recaño, profesor del departamento de Geografia de dicha Universidad e investigador del CED: «El 60\% de los municipios españoles tiene menos de 1.000 habitantes, ocupa el $40 \%$ de la superficie, pero a duras penas concentra el $3 \%$ de población». (Recaño, 2007, p.1).

Son numerosas las iniciativas que se han desarrollado desde diferentes ámbitos, asociaciones, como la Asociación contra la despoblación rural, que trabaja en Aragón, actuando en $200 \mathrm{~km}$ alrededor de Zaragoza, y cuyo objetivo es mantener a los jóvenes en los pueblos, y si es posible asentar nuevas familias ${ }^{6}$. Los foros especializados que reflexionan sobre las causas también son necesarios, como el I Foro Cultura y Medio Rural, organizado por el Ministerio de Educación, Cultura y Deporte, titulado: Procesos para la transformación social, económica y demográfica, que tuvo lugar durante los días 14 y 15 de junio de 2017 en Cerezales del Condado (León). En sus conclusiones redundan en el carácter diverso y heterogéneo que caracteriza al medio rural y que debe traducirse en una respuesta múltiple sin caer en los tópicos que han marcado una visión estereotipada tanto del centro como de la periferia:

«Asistimos a una cierta idealización del mundo rural desde lo urbano, hasta caer con frecuencia en el estereotipo y en cierta estetización -retroruralidad, falsa rusticidad-, que convierte lo rural en símbolo o mera representación, con el subsiguiente vaciamiento de significados y funciones». (AA.VV, 2017, p.1)

Consideramos especialmente interesante el valor que sele concede a la cultura que contribuye y pone en valor el conocimiento del territorio. Apostando por los procesos culturales como dispositivos de transformación, que implicarán tanto al territorio como a las comunidades locales:

«La aproximación del medio rural desde la cultura debe hacerse partiendo del reconocimiento de lo rural como categoría cultural en sí misma. Una cultura que debe ser entendida desde la memoria colectiva y la cohesión social, desde la identidad y la perspectiva territorial y la proyección social del patrimonio". (...) "La cultura aporta precisamente esa capacidad transversal de integración: atraviesa, teje, poliniza, cohesiona, dinamiza, retroalimenta y genera valores añadidos. La cultura como herramienta de desarrollo y transformación eficaz debe surgir y entenderse desde el territorio, conectada a sus necesidades sociales y económicas: una cultura en relación directa e íntima con el lugar, adherida a sus habitantes». (AA.VV, 2017, p.2)

Se reconoce el valor de los proyectos culturales en el medio rural para: «transformar el entorno, fortalecer la comunidad, generar autoestima, desarrollar capacidades y actitu-

6 http://contraladespoblacion.com/ 
des y dinamizar la economía», dichos proyectos deben estar a cargo de un agente cultural, reivindicando la profesionalidad en el sector. La cultura se define, en suma: «como un derecho, un espacio de convivencia y un motor de cambio; ello obliga a pensar menos en las infraestructuras y más en las personas». Queremos destacar también como en las conclusiones de este Foro se afirma que la cultura no funciona como un factor de crecimiento económico en sí mismo, pero si propicia procesos «que favorecen procesos de identidad, motivación, participación, autoestima, resiliencia y cohesión social (...) que sirven para hilvanar la vida social y cuyos efectos transformadores pueden ser de gran magnitud» (AA. VV. 2017, p.4). El II Foro Cultura y Medio Rural. Miradas desde lo contemporáneo, tuvo lugar los días 16 y 17 de mayo de 2018, en Segura de la Sierra, Jaén, en el que se avanzó de forma práctica, a partir de las conclusiones del primer Foro, ya mencionado.

Un documento de referencia que desarrolló algunas de las cuestiones que han sido tratadas en los Foros sobre Cultura y Medio Rural es el Convenio Marco del Consejo de Europa sobre el Valor del Patrimonio Cultural para la Sociedad (Faro, 27 deoctubre de $2005)^{7}$. En su articulado se define, por ejemplo lo que es una comunidad patrimonial: «2b: está compuesta por personas que valoran aspectos específicos de un patrimonio cultural que desean conservar y transmitir a futuras generaciones, en el marco de la actuación de los poderes públicos». Que sería el punto de partida del tema que estamos tratando, pero que también tendrían que tener en cuenta las iniciativas privadas.

En el artículo 10 se trata la dualidad patrimonio cultural y actividad económica estableciendo la necesidad de:

«a) Divulgar el potencial económico del patrimonio cultural y aprovecharlo; b) tener en cuenta la naturaleza e intereses específicos del patrimonio cultural a la hora de trazar las políticas económicas; y c) garantizar que estas políticas respetan la integridad del patrimonio cultural sin poner en entredicho sus valores intrínsecos» ${ }^{8}$

También en los últimos años aparecen iniciativas periódicas que invitan a la vuelta del mundo rural como una segunda oportunidad en un momento en el que las ciudades no ofrecen nada a algunos de sus habitantes. Plataformas como Viuvre rural realizan la intermediación necesaria para aquellas personas que deciden optar por este cambio. Definen su proyecto en el marco del Programa de Desarrollo Rural de Cataluña 2014-2020, han organizado su territorio rural en 11 Grupos de Acción Local (GAL) que cooperan para llevar a cabo proyectos de dinamización social y económica de este territorio. El relevo generacional será un factor clave, pero para que esto se produzca hay que desarrollar iniciativas que sean atractivas para esta nueva población y que no redunden sólo en las facilidades para conseguir una vivienda. Vamos a analizar a continuación algunas experiencias del ámbito rural que han apostado por el arte contemporáneo.

7 https://rm.coe.int/16806a18d3

8 https://rm.coe.int/16806a18d3 


\title{
3. EXPERIENCIAS
}

En términos generales podemos decir que el mayor número de experiencias con continuidad en nuestro país nacen en el siglo XXI algunas de las más longevas, tienen antecedentes marcados por el Land Art:

\begin{abstract}
«El acercamiento del arte al territorio rural en España, se ve imbricado con los movimientos migratorios de la ciudad al campo surgidos en España a partir de mayo del 68, sencillo eco de los realizados en Estados Unidos y Europa en la década de los 60; únicamente cambia de dimensión para adaptarse al contexto cultural, económico, político y social propio de los 60, y por extensión, del último tercio del S. XX, en España se formaron las primeras comunidades migratorias como Arco Iris, en Areyns de Muns, Barcelona; Casa María, en Herrerías, Santander; Ilícitis, en Elche, etc.».(González, 2015, p.144)
\end{abstract}

Contamos con numerosas experiencias que promueven revitalizar el medio rural desde objetivos diversos, por un lado, artistas y colectivos de artistas han trasladado su ámbito de creación al medio rural, por ejemplo el proyecto «Pueblos en arte», en cuyo manifiesto firmado por un colectivo de artistas vemos que su objetivo central es trasladar el arte a zonas rurales prácticamente deshabitadas. La iniciativa surge en 2012 en la localidad zaragozana de Torralba de Ribota. (Gómez, 2018, s.p.)

Evidentemente contribuyen a la despoblación pero en este texto nos vamos a centrar en otro tipo de iniciativas, aquellas que de forma temporal cambian la vida del municipio. Mediante la realización de residencias artísticas puntuales o jornadas, de forma que un acontecimiento estacional dinamiza el resto del año como reclamo turístico. Casos como Fanzara, Genalguacil o Montalbán, que analizaremos a continuación. Estas iniciativas tienen una fuente de financiación tanto pública como privada, siendo más frecuente la primera, aunque el objetivo sería la autofinanciación y se organizan sobre todo bajo el formato de residencias artísticas, cuyo objetivo es la realización de obras que quedarán en el pueblo. Lo que las diferencia unas de otras, como veremos, es el grado de implicación y contacto con el municipio y sus habitantes. Distinguiendo entre aquellas propuestas que dan al artista la oportunidad de crear en contacto con la naturaleza y otras que proponen una mayor presencia e intercambio con los habitantes locales, en este texto nos interesan más las segundas citadas por las implicaciones de sostenibilidad económica que ofrecen al medio rural.

\subsection{Pueblos Museo: el paradigma de Fanzara}

El Museo Inacabado de Arte Urbano (MIAU) de Fanzara (Castellón) es una de las propuestas más visitadas de este tipo en nuestro país, todo tiene lugar en un pequeño municipio de unos escasos trescientos habitantes,que durante cuatro días de verano ve pasar por sus calles más de mil personas al día. Los lugareños ceden los muros de sus casas para 
que artistas de arte urbano de todo el mundo los intervengan y también sus viviendas para alojarlos. «¿Qué es para mí el MIAU? Son cuatro días fantásticos. Una fiesta callejera con muy buen ambiente» contesta María José Gargallo, propietaria de la única tienda de ultramarinos del pueblo y militante convencida de esta propuesta artística «Desde que está esto viene una barbaridad de gente. Excursiones de niños, jubilados...». (Pitarch, 2017)

Todo comenzó en 2004, a partir de un movimiento social que intentaba impedir el vertido de residuos, de ahí, siete años después se pasó al proyecto artístico, creado por Javiér López y Rafa Gascó, habitantes de Fanzara y el artista Miguel Abellán «Pincho», en 2011, con el objetivo de hacer de una localidad condenada al olvido un lienzo para el arte. (Derosas, 2017, p. 100) El MIAU a lo largo de estos ocho años se ha consolidado e institucionalizado sobre todo a partir de 2014. Lo que ha permitido ampliar las actividades no sujetas a la estacionalidad de las intervenciones artísticas. Desde 2015, han pasado más de 3500 estudiantes, las actividades educativas se han llevado también a las aulas de municipios cercanos, de la provincia, como ocurre con el proyecto «Doce artistas, doce escuelas». Es una iniciativa novedosa en nuestro país pero existen otras similares como Museo di Urban Art di Roma (MURo) o Galería de Arte Urbano (GAU) en barrios periféricos de Lisboa, entre otros. (Derosas, 2017, p. 102)

Una vez consolidada la propuesta lo que queda es mantenerla sin que afecte a la población, es decir que siga siendo una propuesta por y para sus visitantes, cuyos efectos redunden en la economía local. «No hay nuevos negocios pero lo importante es que los negocios que había seguramente habrían cerrado de no ser por el desarrollo de este festival». (Pitarch, 2017, s.p.) «Ahora prácticamente todos los días recibimos visitas de gente que viene a disfrutar de las obras» «Lo bueno que tiene este repunte de visitas es que vienen únicamente al pueblo y, por tanto, consumen en nuestros negocios». (Arnau, 2016, s.p.)

\subsection{Residencias artísticas: la visión del artista}

En este texto queríamos tener la visión en primera persona de un artista que ha participado en numerosas residencias durante los últimos cuatro años, agradecemos a Víctor Royás que nos haya transmitido su perspectiva como creador en experiencias que muestran la diversidad de estas residencias9. Víctor Royás, trabaja a partir de la naturaleza, que le permite hablar de lo efímero, por su capacidad transformadora de todo lo que nos rodea, profundizando en su significado simbólico, creando obras a través de la instalación, la fotografía, el video, la acción, etc. en las que cuestiona el comportamiento de la sociedad y su regeneración.

Víctor Royás fue artista residente en Alanís de la Sierra (Sevilla) en 2015, una iniciativa de la corporación local que permitía becar a siete artistas que trabajaron en el

9 Realizamos una entrevista abierta durante el mes de junio de 2018, durante el proceso de documentación del artículo. 
Centro Cultural del pueblo. La temática era libre por lo que se podía trabajar tanto en el espacio público como en el recinto habilitado, según el artista: «El espacio era muy amplio y luminoso, ideal para expandir los materiales y organizar el diseño de la obra», su obra consistió en una acción (Fig. 1) cuyo diseño y confección llevaron los cinco días de residencia y la acción se realizó con el apoyo de La galería de Magdalena en el escaparate de Zuloark, Torre de España (Madrid). La formalización de la obra concluyó en un video que lleva elnombre de Camouflagey que se expuso en la Galería La Casarosa de Málaga. «Con Camouflage trabajé la idea de ficción y poder, que nos lleva a perder la conexión con la naturaleza y con nuestra propia esencia. Cuestionándome el concepto de 'realidad'». La residencia incluía visitas a los hitos culturales del pueblo y excursiones a sus alrededores pero «no hubo presentación de las piezas a los vecinos del municipio, por lo tanto se echaba de menos ese contacto con el espectador» que si existe en otros proyectos.

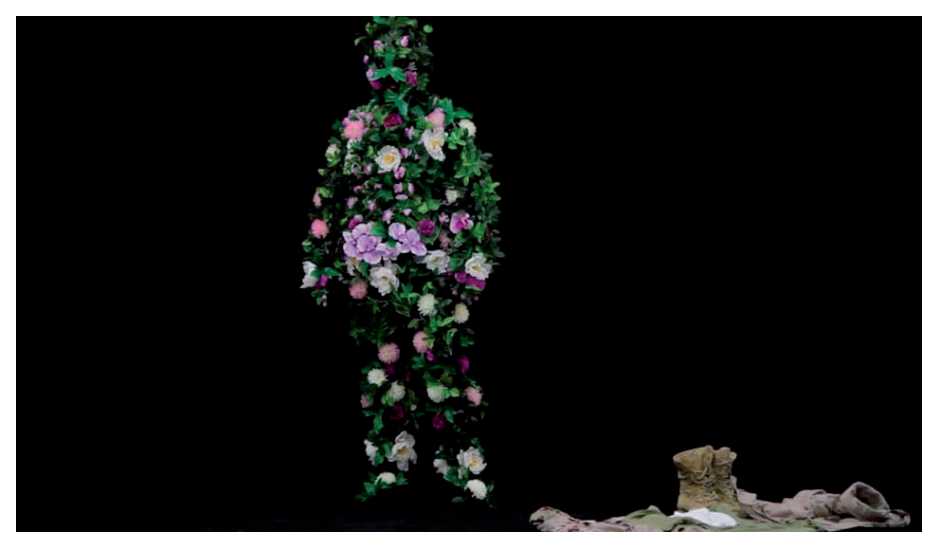

Figura 1. Camouflage, Víctor Royás, fotografía facilitada por el autor.

Otra experiencia es la beca de producción Kaldarte, Caldas de Reis (Pontevedra) en 2016, donde reside durante cinco días, en este caso está enfocado $100 \%$ al arte urbano o arte efímero, todas las intervenciones se realizan en el espacio público del municipio. Kaldarte, cuenta con una sólida experiencia desde su inicio en 1998. Aunque se pernocta en un hotel, se dispone de espacios donde realizar las piezas, la organización cubre todo tipo de necesidades técnicas que solicite el artista. En esta residencia Víctor Royás realizó una pegada de carteles de la serie, Scream of Flowers, que previamente había impreso en Madrid (Fig. 2) «En estas piezas se cuestiona la libertad de expresión en un país democrático, creando paralelismos con el desarrollo natural de la vegetación». Para el desarrollo de la obra pidió la colaboración de asociaciones y vecinos que fueron entrevistados por el artista. En este caso hubo inauguración de las obras realizadas con las autoridades y la vecindad, destacando el «apoyo económico de producción, viaje y estancia, donde incluye también la manutención y en mi estancia se realizó también una salida a Vigo para celebrar la finalización de los proyectos». 


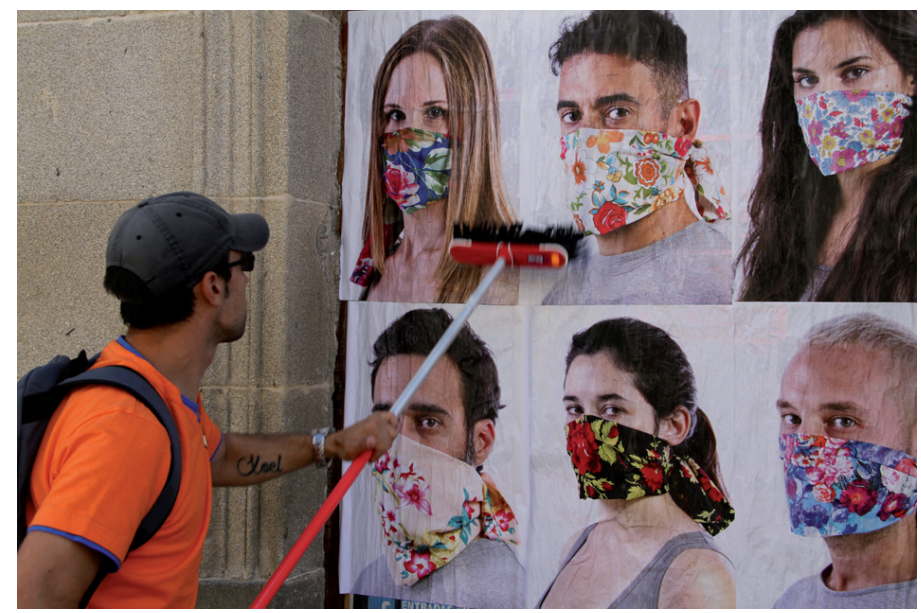

Figura 2. Scream of Flowers, Víctor Royás, fotografía facilitada por el autor.

Una de sus últimas residencias ha tenido lugar en Cañete de la Torre (Córdoba) del 3 al 6 de mayo de 2018 para participar en una convocatoria nacional e internacional que lleva el nombre de Calles en Flor, «tras la selección,la organización adjudica según el trabajo por dossier, una ubicación. Posteriormente a la selección se da un plazo de unos 20 días para presentar un proyecto para el enclave que se envía fotográficamente. En mi caso me adjudicaron el Parque de Andalucía, en el cual hay un monolito In Memoriam de las Víctimas de Género».

Los artistas seleccionados deben instalar sus obras en los tres días que dura el evento y presentarlas a las autoridades provinciales y vecinos, «la visita tiene un carácter solemne y festivo acompañando al cortejo, la banda municipal». Hay un apoyo logístico y económico importante para la realización de las piezas. Aparte de la beca se cubren los gastos de manutención y alojamiento en el hotel del pueblo. «Destacaría de Calles en Flor la implicación del pueblo en los procesos de realización de las piezas, el apoyo de asociaciones, en mi caso la asociación de víctimas de género y la generosidad de los vecinos».

Observamos grandes diferencias entre unas y otras residencias, todas están financiadas por las corporaciones locales pero su incidencia en el municipio y en sus habitantes es variable, por todo esto podemos concluir que aquellas residencias que sólo ofrecen el ámbito de trabajo tendrán una dependencia directa con las partidas económicas destinadas a tal efecto e incluso asociadas a las corporaciones locales. Lo deseable sería que ya que se invierte redunde en el propio ámbito desde dos puntos de vista, conservando la obra, ya que la colección puede convertirse en un reclamo turístico, como veremos y fomentando el intercambio con la población, formándola y familiarizándola con la creación contemporánea para que haga suyas estas experiencias. 


\subsection{La recuperación de la memoria local. Isidro López Aparicio: Encuentros de Arte Genalguacil, 2016}

Los Encuentros de Arte de Genalguacil (Málaga) llevan, con periodicidad bianual, a artistas de todo el mundo a este pequeño municipio de la serranía de Ronda, que no supera los 500 habitantes. Se iniciaron en 1994, pero será a partir de su edición duodécima, de 2015, cuando tengan una mayor continuidad. El Ayuntamiento de la villa se encarga de costear la manutención junto al alojamiento de los artistas, cuyos proyectos han sido elegidos. Lo interesante de este encuentro es que las obras que realizan los artistas durante dos semanas permanecen en el pueblo, que se ha convertido en un Museo al aire libre que atesora más de 200 creaciones $^{10}$. La artista gallega Carolina Cruz cuando llegó en 2012 pensó «Genalguacil es un lienzo en blanco que está a la espera de que le den color» (Mora, 2012) Aunque no se recogen los datos de forma sistemática, según nos confirmaron en el Ayuntamiento, los Encuentros de Arte han dinamizado la afluencia de visitantes a un municipio que por su propia orografía dificultaba el acceso. Por estemotivo han decidido que aunque los Encuentros sean bianuales, en los años pares, que en los impares se celebre una Semana Cultural relacionada con el arte contemporáneo.

Para este artículo queríamos contar con el testimonio de Isidro López Aparicio, artista seleccionado en 2016 (Edición XIII, desde el 31 de julio al 14 de agosto) al que agradecemos el relato de lo que fue esta experiencia ${ }^{11}$. Isidro López- Aparicio es un artista multidisciplinar, su compromiso personal impregna sus proyectos artísticos que han girado en torno a la denuncia social. Le interesan todos los temas que afectan a las personas, sus conflictos y sus relaciones, tratando siempre de captar los distintos puntos de vista. Él cree, por tanto, que el Arte puede ser útil para la sociedad, al menos en cuanto a provocar una reflexión en el observador (Quirosa, Luque, 2015, pp. 253-254) «Trabaja con objetos, los rescata, propone arqueologías de lo cotidiano para activar y revisar nuestra memoria cercana»12.

«Mi paso por los Encuentros de Arte en Genalguacil es uno de los tantos ejemplos en los que se pone en práctica mi aproximación a la creación artística desde un acercamiento humano y holístico. El entendimiento que defiendo del arte público va más allá de aquel que se define como: el que se muestra en un espacio público, sino aquel que es tejido con lo social de manera que por la participación activa del contexto humano se construye una propuesta en la que cuando se muestra (incluso indistintamente de la tipología del espacio) adquiere el carácter público».

El artista nos cuenta como en este tipo de convocatorias artísticas presenta un proceso de creación y no una obra en sí:

10 http://www.genalguacil.es/es/Arte_y_cultura/Encuentros_de_Arte/Presentacion/

11 Realizamos una entrevista abierta durante el mes de julio de 2018 , durante el proceso de documentación del artículo.

12 http://www.artium.org/es/explora/exposiciones/item/60300-isidro-lopez-aparicio-la-memoria-de-losobjetos-praxis 
«Esto genera muchos interrogantes en muchos casos pero para mí ha sido y es una manera lógica de construir dicha obra de manera coherente dentro del contexto. Esto implica muchos riesgos pues no sabes cuál será la manera de solucionarlo, y por otro lado conlleva el haberse formado profesionalmente a un gran nivel de forma que pueda tener uno la seguridad para encontrar resoluciones conceptuales, formales, técnicas, sociales...».

Su proceso comienza con una investigación previa de la zona, el Valle del Genal: «desde lo histórico a lo antropológico, pasando por lo folklórico, la agricultura... y con respecto a lo artístico un análisis de las propuestas artísticas llevadas a cabo y sus implicaciones», teniendo en cuenta que la obra quedará expuesta en el municipio de forma permanente. También considera esencial en el inicio del proceso conocer a los vecinos del pueblo: «utilizando la excusa de los objetos, preguntándoles por aquellos de los que pueden despojarse y que estarían dispuestos a aportar para que forme parte de una obra artística» (Fig. 3). Nos parece especialmente interesante esta parte en la que el artista entra en contacto con las mujeres que: «comienzan a contar sus vidas y compartir también aquellos objetos e historias que son verdaderamente significativas para ellas». De esta forma se rueda el documental específico de las mujeres del Genal que será una pieza fundamental de la intervención, es la primera vez que estas mujeres son las protagonistas: «la forma de mostrar el video alrededor de una mesa de camilla y con las sillas que ellas mismas han donado para la obra (...) de esta forma el propio pueblo entra en el Museo».

«Me llegan comentarios de vecinos como por primera vez ven a las mujeres del pueblo al Museo a sentarse y echar un rato viendo una obra de arte en las que ellas son las protagonistas y como cuando llegan sus familiares los llevan a que lo vean. Y claro esta obra es una parte que se completa con la escultura que hay en la calle y que obligatoriamente se ven forzados a verla para comprender todo el proceso de cómo se generó y en la que el pueblo es participe activo».

Figura 3. Isidro López-Aparicio, Encuentros de Arte Genalguacil, 2016. Fotografía facilitada por el autor.

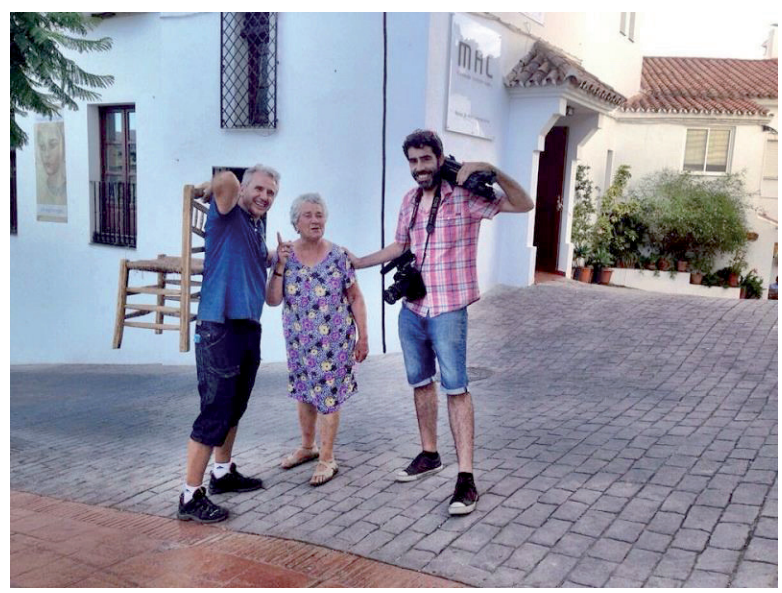


En una segunda fase Isidro López Aparicio estudia los objetos que les han sido entregados por los habitantes, es en este momento en el que decide: «hacer un arco volado en una calle que da entrada al pueblo y que permite dar dos visiones una hacia dentro del pueblo, dando la bienvenida y otra hacia la montaña despidiéndose de los visitantes». Es un lugar en el que ningún artista había trabajado aún ya que parece poco idóneo por el fuerte viento que lo azota, «pero a pesar de la dificultades tengo claro el que debo de hacer el esfuerzo y asumir las dificultades pues esta obra la planteo con dos etapas fundamentales para cumplir los múltiples objetivos que me planteo cubrir».

La elección de la ubicación está determinada también por el uso que harán los habitantes de Genalguacil, ya que sus paredes se blanquearán cada año como es tradición pidiendo que, «también se blanquee la escultura, que si ya era del pueblo pues se había hecho con sus objetos ahora se da un nuevo paso de forma que la integre en el pueblo». La otra razón es que esta calle forma parte del recorrido de la procesión del Santo de Genalguacil (Figs. 4, 5)

«Mi intención es que dicho arco (símbolo de los habitantes del Valle del Genal y sus memorias) orle el paso de su Santo, de forma que la escultura se convierte en el escenario en el que el Arte Contemporáneo y la tradición conviven en total armonía, dentro de ese gran performance social como es la procesión».

Figura 4. Isidro LópezAparicio, Encuentros de Arte Genalguacil, 2016. Fotografía facilitada por el autor.

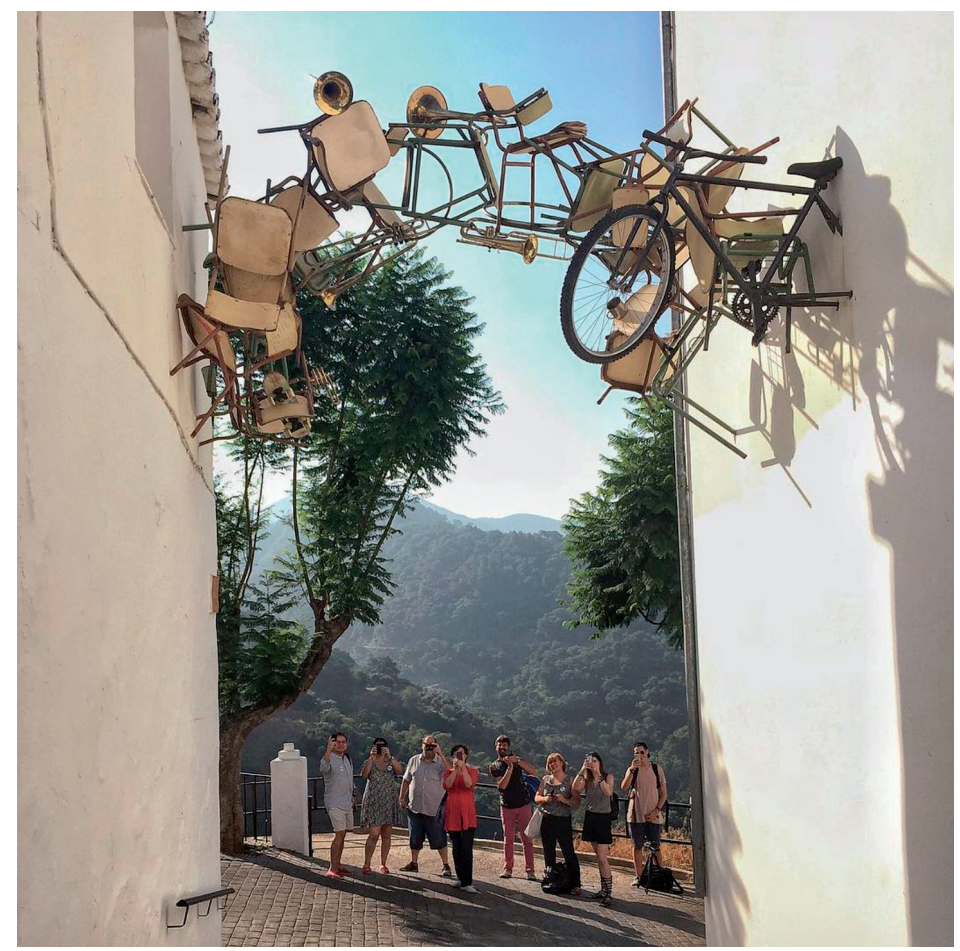


Figura 5. Isidro López- Aparicio, Encuentros de Arte Genalguacil, 2016. Fotografía facilitada por el autor.

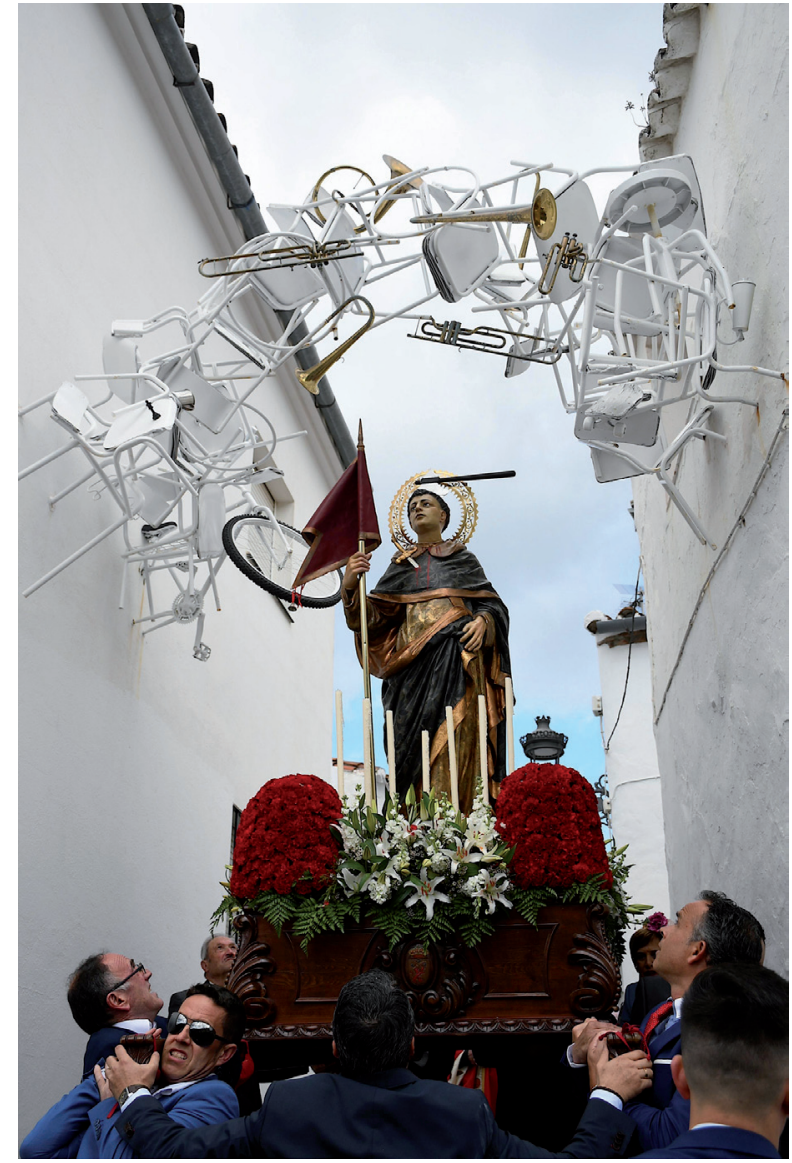

\subsection{La visión de los coordinadores. Intervenir las calles: Z Montalbán (Córdoba)}

La puesta en marcha y la continuidad de un evento artístico requiere un empeño personal a gran escala por parte de sus promotores y la colaboración de los agentes locales. Es paradigmático el caso de la provincia de Córdoba, un referente en la puesta en valor de su medio rural a través de citas que a lo largo de todo el año apuestan por integrar el arte contemporáneo y la periferia rural, el propio nombre que los aúna así lo contempla «Periféricos», entre los que se encuentran, Aptitudes (La Rambla) desde 1997, con intervenciones fotográficas en vallas publicitarias o street art, etc. Dmencia (Doña Mencía) desde 1996, dedicado a la experimentación expositiva, etc. Las líneas queestablecían la periferia se diluyen con el avance de las comunicaciones y las nuevas tecnologías, para Javier Flores «El panorama actual tienen a ser un archipiélago de ideas (...) existe un amplio conjunto de proyectos susceptibles de formar una red de encuentro, diálogo y conexión que no corresponde a un modelo preestablecido» (Flores, 2010, p.184) 
Z, Jornadas de arte contemporáneo de Montalbán (Córdoba) nace en 2012 bajo la coordinación de Demetrio Salces y Rafael Jiménez, como plataforma y espacio de experimentación creativa en torno al arte y la cultura actuales en el medio rural, a través de tres líneas de trabajo: acción, difusión y formación. En las Jornadas se desarrollan diferentes actividades en torno al arte y cultura contemporáneas en sus múltiples manifestaciones, ocupando diferentes espacios culturales y urbanos del municipio y en relación e interacción con sus habitantes.

Queríamos contar en este texto con la visión de sus Coordinadores para lo que nos pusimos en contacto con el artista Rafael Jiménez ${ }^{13}$ que amablemente nos transmitió junto a Demetrio Salces, también artista, sus impresiones al frente de Z de Montalbán (Córdoba) que llevará a cabo en 2018 su séptima edición ${ }^{14}$.

En palabras de Demetrio Salces y Rafael Jiménez:

«El objetivo de Z, desde un carácter humilde, realista y con una escala de acción muy medida en la localidad, es la de poder ofrecer diferentes puntos de vista del arte y cultura contemporánea del presente de la forma más cercana sin perder calidad o rigor, esto es naturalizando y estableciendo puntos de contacto entre artista, obra y público lo más inclusivos posible, ya sea desde la propia programación hasta la participación directa relacionando a agentes culturales con asociaciones y colectivos locales». «Nuestro interés es presentar el arte contemporáneo fuera de tópicos, y de una forma didáctica que sea lúcida a la vez que lúdica sin caer en el error de plantear la cultura o la creación como mero entretenimiento o espectáculo».

El proyecto cuenta exclusivamente con financiación pública a través del Ayuntamiento de la localidad y la Fundación de Artes Plásticas Rafael Botí (Córdoba) a través del programa específico Periféricos. Reivindica algo que nos parece esencial en la gestión de la cultura, «la dignificación del trabajo del creador y la progresiva profesionalización de la gestión». Su apuesta por la reivindicación de la periferia al igual que otras citas de la provincia quiere: «poco a poco ampliar las posibilidades de trabajo, colaboración y proyección para creadores y proyectos de cercanía» lo que lleva a «cuidar mucho la selección de artistas que invitamos en cada edición y manejar perfiles que entendemos que son interesantes para la interacción con el medio».

Rafael Jiménez y Demetrio Salces destacan como:

«No hay que olvidar que el trasiego de agentes, público, participantes y la actividad generada durante las jornadas supone un impacto económico valorado por los negocios locales, con los que de la misma forma trabajamos en la producción de exposiciones o proyectos desde el propio pueblo tratando de no externalizar fuera de la localidad ese tipo de servicios».

13 Realizamos una entrevista abierta durante el mes de julio de 2018, durante el proceso de documentación del artículo.

14 http://zjornadasdearte.blogspot.com/p/z.html 
Desde el inicio de las jornadas en 2012 el interés de la población hacia el arte contemporáneo ha crecido convirtiéndose en algo cotidiano para sus habitantes la presencia de artistas y obras (Figs. 6-8) Sus coordinadores nos confirman como estas jornadas son capaces de:

«Generar nuevas lecturas desde el presente hacia espacios y señas de la idiosincrasia de la localidad (caminar desde una visión global hasta un localismo reconocible transitando diferentes niveles de acción y lectura) proporciona nuevas señas de identidad para los vecinos, respetando de esta forma las tradiciones y formas de ver ya existentes y confrontándolas con el presente desde la suma de identidades».

Sin duda las jornadas Z de Montalbán cumplen las características necesarias por las que el arte contemporáneo se convierte en un agente dinamizador desde un punto de vista social y económico del medio rural.

Figura 6. Z Montalbán (Córdoba) fotografía facilitada por Rafael Jiménez.

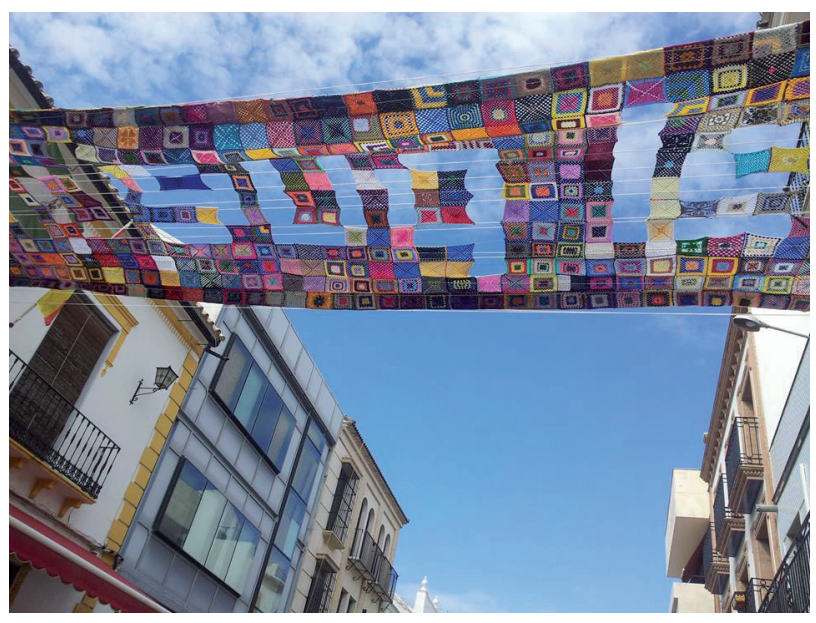

Figura 7. Z Montalbán (Córdoba) fotografía facilitada por Rafael Jiménez.

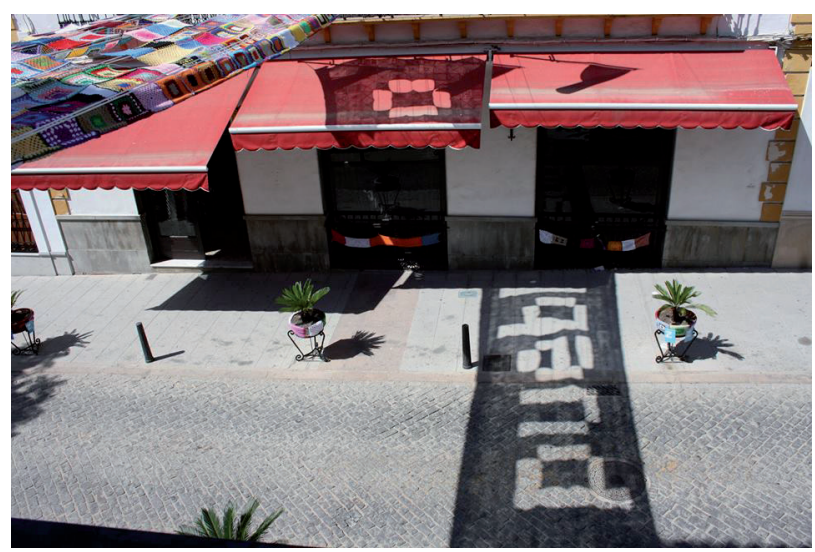


Figura 8. Z Montalbán (Córdoba) fotografía facilitada por Rafael Jiménez.

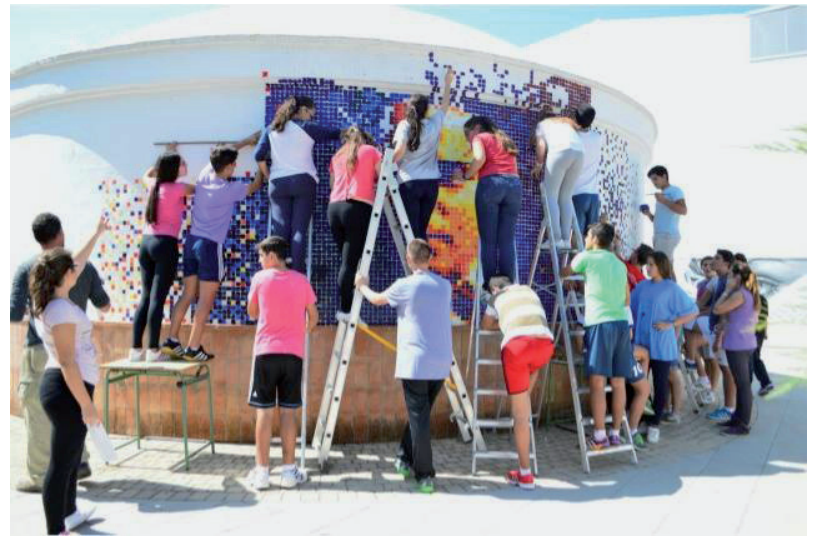

\section{CONCLUSIONES}

Desde comienzos del siglo XXI proliferan en nuestro país iniciativas que de forma estacional llevan el arte contemporáneo a nuestros municipios, los medios, como hemos visto, son muy diversos y van desde la invitación a artistas que cambiarán su taller por otros paisajes, sin que la obra permanezca en el municipio a programas que pretenden crear una nueva identidad en estas localidades a través de intervenciones.

Teniendo en cuenta que se trata en un gran número de casos de municipios muy pequeños, que rondan los 300-500 habitantes, estas actividades bien entendidas pueden generar un motor de cambio social y económico. Casos como Fanzara o Genalguacil lo evidencian, aunque a día de hoy no hay un registro cuantitativo del número de visitantes que reciben, la vida en estos municipios ha cambiado sensiblemente y los ha situado como enclaves turísticos de forma distintiva frente a otras localidades, ya que el producto o la experiencia que ofrecen es diferente.

Lo importante es que esta inyección económica que permite ralentizar su despoblamiento revierta en otros ámbitos sin que se desvirtúe el objetivo inicial, estas iniciativas deben ser por y para el pueblo y no enfocadas a los agentes externos, como nos muestra la experiencia de los últimos años, ese es el reto. Uno de los problemas que tienen que superar es la estacionalidad, por eso una vez consolidado el proyecto se tiende a incorporar un programa de tipo didáctico que permite el acercamiento a la población más allá de las residencias artísticas o ejecución de las obras, creando así un recurso sostenible todo el año.

No podemos olvidar que un valor esencial de estos proyectos es el formativo, experiencias como $\mathrm{Z}$ en Montalbán ponen de manifiesto que la población del municipio se interesa y se familiariza con el arte contemporáneo desde edades tempranas. Su desarrollo en núcleos periféricos será necesario ya que para algunos estratos de la población será su primer contacto directo con la creación artística, incluso colaborarán y participarán de 
forma activa en el proceso. Iniciativas como las desarrolladas en Córdoba son necesarias, ya que además de lo dicho permiten la profesionalización del sector y promueven un cambio en los agentes sociales.

Las experiencias analizadas ponen de manifiesto como una pequeña inversión económica en el ámbito de la cultura, mejora la vida en nuestros pequeños municipios, los vuelve a situar en el mapa, les da una segunda oportunidad para mostrar con orgullo sus señas de identidad tanto del pasado como del presente. 


\section{BIBLIOGRAFÍA}

AA.VV., Cultura y ciudadanía. Documentos. Conclusiones del I Foro Cultura y Medio Rural. Procesos para la transformación social, económica y demográfica. Ministerio de Educación, Cultura y Deporte. 2017.

Achiaga, P. «Eugenio Ampudia.’Todos los problemas actuales implican algún fallo en la comunicación», El Cultural, 7 de febrero de 2015. https://www.elcultural.com/noticias/buenos-dias/Eugenio-Ampudia/7351

Arnau, J., «Overbooking” turístico en Fanzara», El Mundo, 19 de agosto de 2016. http://www.elmundo.es/comunidad-valenciana/castellon/2016/08/19/57b6e5fee5fd ea4d7e8b45ee.html

Bourriaud, N., Estética relacional. Argentina, Adriana Hidalgo Editora. 2008.

Careri, F.,Walkscapes. El andar como práctica estética. Barcelona, Gustavo Gili, 2014.

Derosas Contreras, D., «Museo Inacabado de Arte Urbano (MIAU): Musealización del paisaje urbano». Revista de Didácticas Específicas, n 17, 2017, pp. 99-108.

Díaz Guardiola, J., «Eugenio Ampudia: El silencio, hoy, es una forma de tomar partido» .ABC cultural 11 de marzo de 2015.

https://www.abc.es/cultura/cultural/20150311/abci-entrevista-eugenio-ampudia-201503111739.html

Espejo, B., «Isidoro Valcárcel y Dora García: Preferiría sí hacerlo», El País, 16 de abril 2018. https://elpais.com/cultura/2018/04/12/babelia/1523544312_107232.html

Feilden B. M, Jokilehto J.,Manual para el manejo de los sitios del Patrimonio Cultural Mundial. Roma. ICCROM, UNESCO, Centro del Patrimonio Mundial (WHC) e ICOMOS, 2003.

Flores, J., «Periféricos. Arte contemporáneo en la provincia de Córdoba», Periférica internacional. Revista para el análisis de la cultura y el territorio, 11, 2010, pp.177-187.

Gómez Ruíz, L., «Pueblos casi deshabitados que reviven gracias al arte y la cultura». La Vanguardia. 13 de abril de 2018.

http://www.lavanguardia.com/cultura/20180413/442488158500/pueblos-ruralesreviven-arte.html

González Fernández, M.,«Recuperación del patrimonio mediante prácticas artísticas contemporáneas». LIÑNO Revista Anual de Historia del Arte, 21, 2015 pp.143-152.

López Aparicio, I., Arte político y compromiso social. El Arte como transformación creativa de conflictos. Murcia, CENDEAC, 2016.

Lowenthal D., El pasado es un país extraño. Madrid, Akal, 2010.

Mora Caballero Antonio J., «Genalguacil. Un pueblo con arte». El País. 6 de agosto de 2012.https://elpais.com/ccaa/2012/08/06/andalucia/1344280005_823301.html

Parcerisas, P., Conceptualismo(s) poéticos, políticos y periféricos. En torno al arte conceptual en España, 1964-1980. Madrid, Akal, 2007.

Pastor Valls, M.T. «M.I.A.U. (Fanzara) una propuesta social. Historia, materiales y conservación». Revista Ge-conservación, 10, 2016, pp.135-145. 
Pitarch, M., «MIAU: un maná en formato arte para los 280 habitantes de Fanzara». El País. 8 de julio de 2017 https://elpais.com/ccaa/2017/07/08/valencia/1499524728_562968.html

Quirosa García, V. y Luque Rodrigo, L., «Arte útil para la sociedad. Consideraciones entorno a seis artistas del siglo XXI en España». De Arte, 14, 2015., pp. 253-254.

Recaño, J. La sostenibilidad demográfica de la España vacía.PerspectivesDemogràfiques, 7, julio 2007. Centre d'EstudisDemogràfics y UniversitatAutònoma de Barcelona. http://ced.uab.cat/PD/PerspectivesDemografiques_007_CAST.pdf

Rivière G.H.,«Definición evolutiva del ecomuseo», Revista Museum, 148, vol. 37., 1985, pp. 182-184. 